\title{
The Kinetic Family Drawing Test for Evaluating Interpersonal Dynamics in Families: A Case Study
}

\author{
Anupama Bajaj $^{1}$, J.M. Asgarali Patel ${ }^{2}$, Anuradha Kabra ${ }^{3}$ \\ ${ }^{1}$ Research Scholar, Department of Psychology, Annamalai University, Annamalainagar-608002, ${ }^{2}$ Associate \\ Professor \& Head in-Charge, Department of Psychology, Annamalai University, Annamalainagar-608002, \\ ${ }^{3}$ Consultant Clinical Psychologist \& Art Based Therapist, Department of Psychiatry, Manipal Hospital, \\ Bengaluru-560008
}

\begin{abstract}
The purpose of this study is to evaluate interpersonal dynamics through Kinetic Family Drawing (KFD). Art Based Therapy (ABT) can be used in situations where talk therapies and counselling skills are least effective. It is especially helpful for people in crisis and those who are experiencing extreme psychological states. This case study has employed The Kinetic Family Drawing (KFD) to diagnose and treat 62 years old married woman with complaints of sadness, uncertainty \& fear. This ABT program comprised of 3 sessions in which the Burns and Kaufman's (1972) Kinetic Family Drawing (KFD) was used ${ }^{4}$. In each session the client was made to draw a picture on a sheet of paper $(8.5 " \times 11 "){ }^{4}$. The picture drawn by the client was subsequently analysed using focal points and the results were discussed with the client. The outcome of the present study helped the client to get insight into her problem and regain her self-confidence and improve relationship with her family members.
\end{abstract}

Keywords: Art Based Therapy, Kinetic Family drawing, Case study, Insight building, mental health, selfconfidence, family dynamics.

\section{Introduction}

Diverse art forms have been an essential part of human life since primeval period. For generations art have been put into practice in every culture to articulate, generate, and communicate the needs, desires, and ideas among human beings. Arts-Based Therapy (ABT) is a term coined by World Centre for Creative Learning (WCCL) Foundation in 2001 to represent the use of multiple art forms and their combinations in therapy. ${ }^{3}$

The formal practice of art therapy has its origins in the mid-20th century in Europe, with the coining of the term being attributed to British artist Adrian Hill in 1942. By working to bring the conscious, unconscious and subconscious into expressive and tangible forms, the creative processes involved in art therapy have been praised for their ability to encourage personal growth, mindfulness, and self-discovery. The client creates art images 'to help express who they are, to express feelings and ideas, and to enhance life through self-expression'2. Analysing the drawings of the client enables health professionals understand wide spectrum of client's mental health issues ${ }^{1}$.

The purpose of the present study: The main objective of this study is to enable the client to release her feelings, emotions, and worries and help her understand the family dynamics and improve relationship with family members.

\section{Method}

Sample: The client is a 62-year-old married woman (housewife) with prolonged history of psychological and familial issues. The client has a past history of psychological treatment which did not help her fully.

Tool: The Burns and Kaufman's Kinetic Family Drawings (KFD) technique was used for the present study ${ }^{4}$. The KFD was created as an extension of the Family Drawing Test ${ }^{4}$. The directions given to the client is stated as follows: "Draw a picture of everyone in your family, including you, doing something, some kind of 
action. Try to draw whole people, not cartoon or stick people $4 \& 5$." This picture is meant to elicit the person's attitudes toward his or her family and the overall family dynamics ${ }^{4}$.

Procedure: The kinetic family drawing was administered on the client.In each session the client was instructed to a draw picture based on KFD instructions on a sheet of paper $(8.5 " x 11 ")^{5}$. The pictures were analysed and discussed using focal points.

$\mathbf{1}^{\text {st }}$ Session: After the intake session, $1^{\text {st }}$ session of art therapy was planned. "The client was instructed to draw her present family doing something". 5

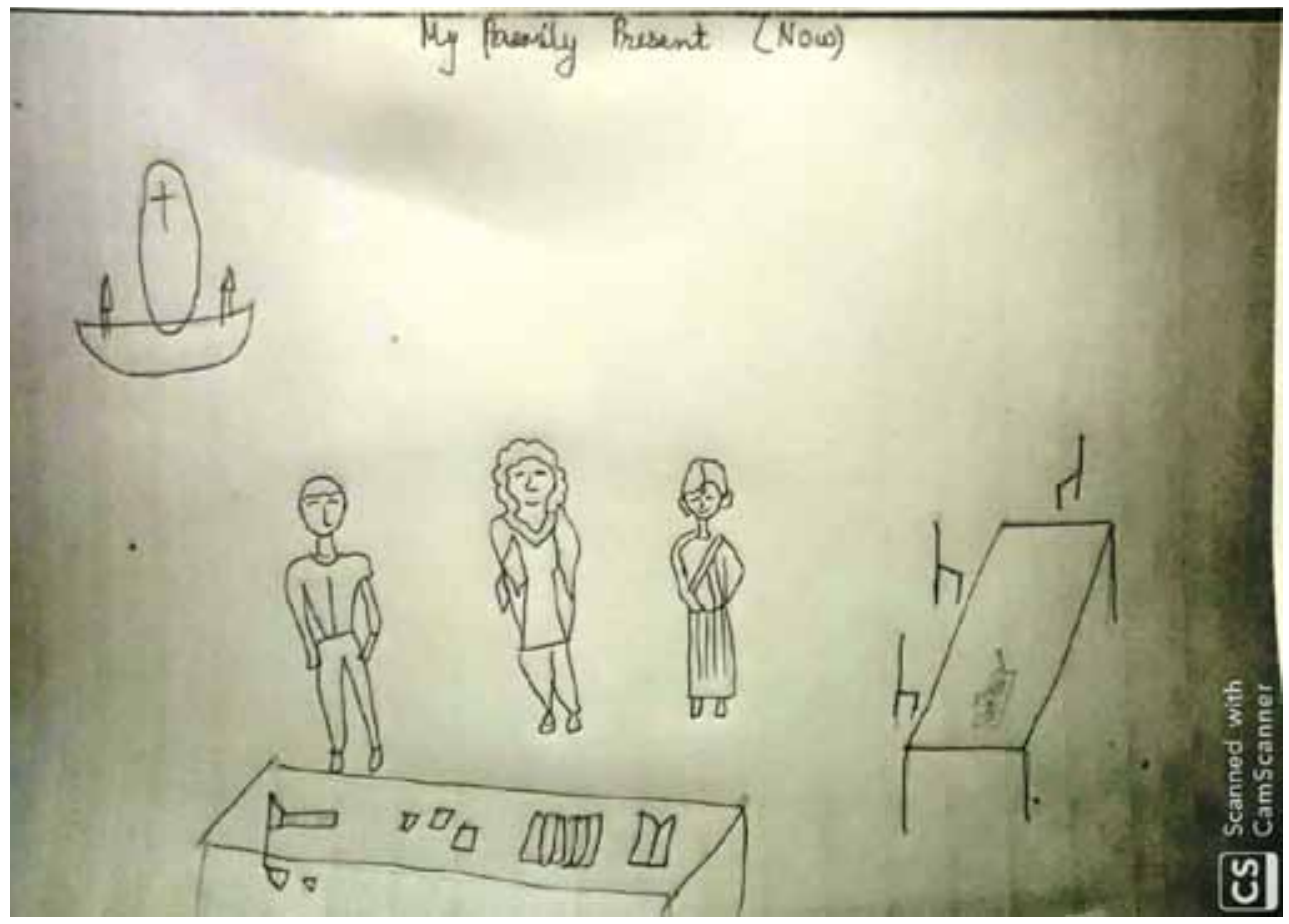

Drawing 1: 1st session (November 2019)

Session 1: Analysis of focal points: The client is a 62-year-old married woman (housewife) with prolonged history of psychological and familial issues. The family consisted of the client, her spouse (72, retired from business) and daughter (31, working as project manager). The client has a past history of psychological treatment which did not help her fully. The client has certain issues with her family members, and she is not happy with her present situation. The client appeared sad as her daughter has been facing issues in her marriage. The client had to leave her job to help her daughter.

In figure 1-seven characteristics dominated KFDLining on the bottom of the paper, Underlining, X's, light (candle), placement of figure, Size of figures, elevated figure.

"Lining on the bottom of the paper is typical of children who feel instability in the home and are trying to maintain stability by creating a very solid foundation" ${ }^{4}$. The client had drawn a table ${ }^{10}$ beneath her leg, which indicates that she feels instability in her house and she might be looking for some ways to create stability.

Underlining- "the instability reflected by such lining is focus on an individual figure, in which case only that figure will be heavily underlined. Underlining at the base of the drawing reflects the extreme tension in the family, unstable relationship ${ }^{10}$ symbol of that which links to source of nurturance" 6 . Here the table drawn beneath the leg of the client's spouse indicated underlining. May be the client's spouse is undergoing lots of stress and instability ${ }^{10}$, looking for attention and his need for nurturance is high. The client stated that she is not able to provide enough support to thespouse. 
$\mathrm{X}$ 's indicates "the person is struggling for love and warmth" 4 . The client has drawn two candles (energy) with the cross $^{11}$ in the centre suggesting the need for love, warmth and direction in her life. There may be possibility that the client is inhabited by a strong conscience or superego. The client has also drawn source of light (Energy), showing"There is very little warmth and love in the family. The person must be of obsessive need for warmth and love." ${ }^{4}$ Elevated figure"A dominant person will be placed high above the rest of the figures" $"$. The client has drawn her daughter elevated which shows that daughter has dominant qualities.

"The placement and size of the figures in the drawing shows, largefigures, perceptions of power or aggressiveness and small drawing of the self indicates poor self-concept and feelings of insignificance ${ }^{8}$. When an individual draws the self-next to a significant other, it may indicate that the person likes that individual, wishes to be closer, and wants more attention."4 The client drew herself, daughter and her spouse at distance from each other. The daughter has been drawn the largest, may indicate that she is powerful and aggressive in the family. The spouse is the smallest relative to family members suggesting that he may have feeling of insignificance and poor self-concept ${ }^{10 \& 11}$. The client has drawn herself next to her daughter suggesting that she is close to her and helping her deal with marital difficulties.

$2^{\text {nd }}$ Session: The first session of KFD was an eye opener for the client. The client decided to comeagain as the insights from the first session were very helpful. This session was approximately three months after the first session.

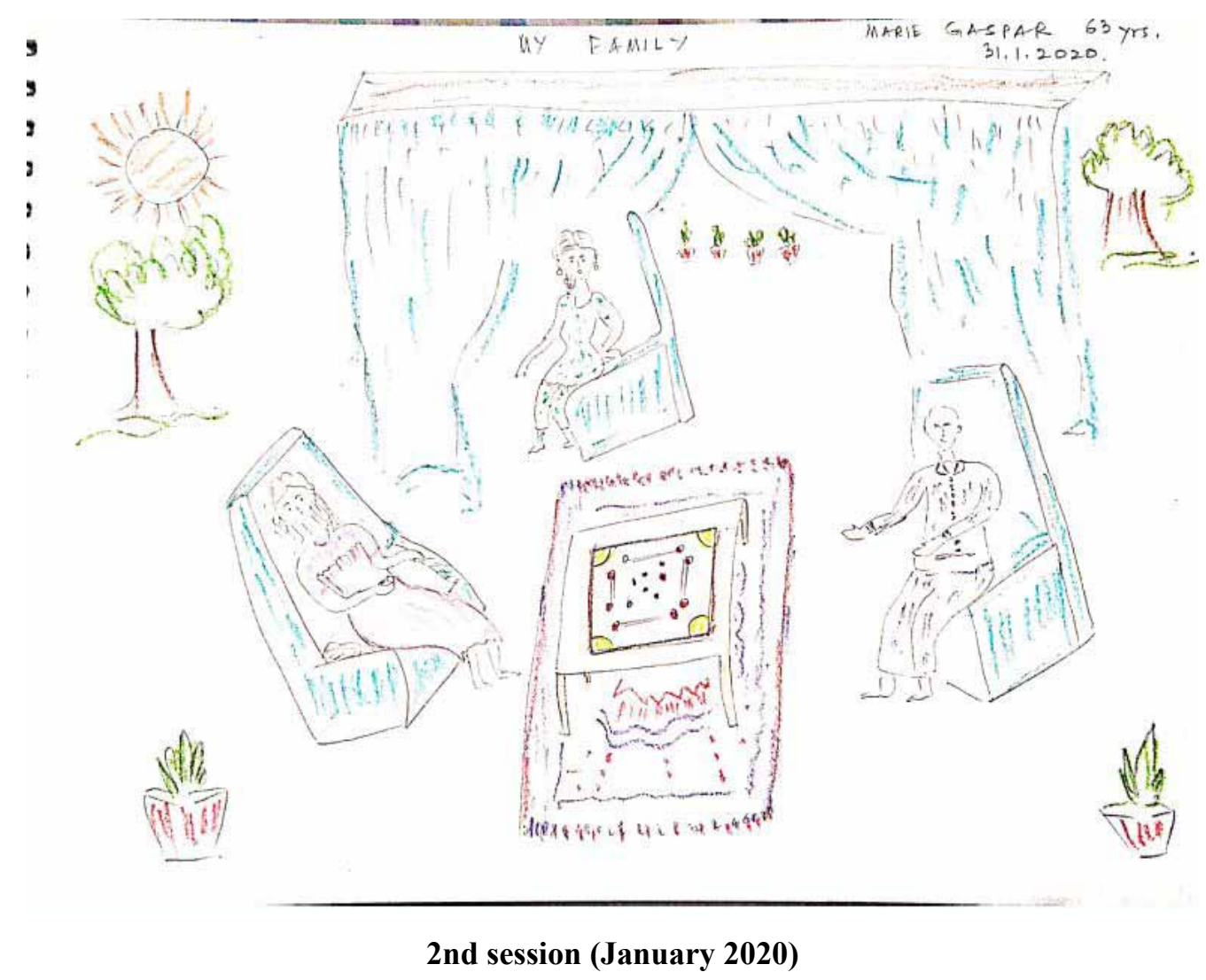

Session 2: Analysis of focal points: The second KFD (figure 2) defines the family dynamics and progression of therapy. The client is feeling much calmer \& positive. The conflict with her daughter \& spouse is still present as she has encapsulated them in the drawing. "Encapsulation is the encircling of individual family member, with an element of tension in the family. Theoretically it is thought to reflect isolation, rejection,threat and adjustment", $4,5 \& 6$

The client depicts her daughter and spouse playing carom enclosed by large chair. Throughout the therapy the client was feeling unhappy about her daughter's life and she was looking forward to get some help in that 
direction. In drawing two the need for love and warmth has taken a form of $\operatorname{sun}^{12}$ (energy) instead of candles ${ }^{5}$. There is no $\mathrm{X}$ drawn in the second drawing suggesting that the struggle for love and warmth was resolved by the client by getting into religious practices. Placement of figures has changed; the client drew herself, daughter and her spouse around the dinner table which shows improvement in relationship. Daughter is still placed as an elevated figure. There is no line drawn at the bottom of the page suggesting that the client need for support has been taken care either by the family member or by the counsellor. Compartmentalization is "as an intentional separation of family figures through lining. This probably reflects the importance of boundaries and beginning of separation for the people living in the family" 4 . The client has used compartmentalization by separating the family figures through curtain lining. The client is trying to create a boundary between herself and her daughter. Some new elements like flowers and trees were added. "Flowers\& trees represent love of beauty and the growth process" $4 \& 5$. It symbolizes client's religious and spiritual growth. Religious practises have made the client emotionally stable to handle her conflicts ${ }^{11}$. Table at the centre of the picture depicts obsessive need for attention and food." 10 This may suggest the client is not getting enough attention and acknowledgement from her spouse and daughter. In summary the second drawing reinforces several basic themes present in the first drawing but it also demonstrates a clear improvement, which was supported by client's ve

3rd Session: After the second session of KFD the client has started looking at life with a different perspective. The client is feeling calmer \& positive.

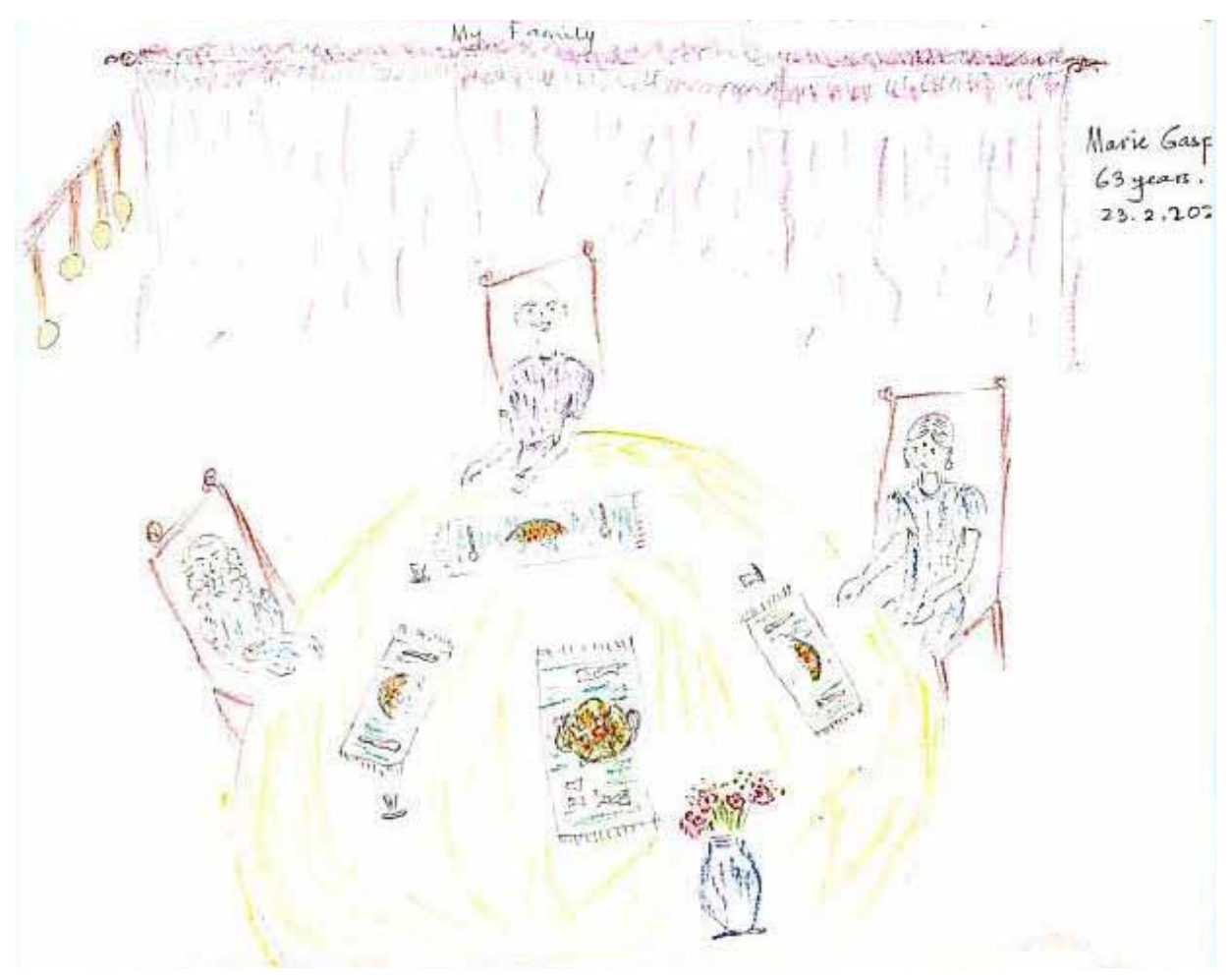

3rd session (February 2020)

Session 3: Analysis of focal points: In drawing three, the client presents herself united with the family. The conflict with her daughter $\&$ spouse is still present as there is a need to encapsulate. The client depicts her daughter and spouse eating food enclosed by chair. The compartmentalization is removed, indicating the closeness \& togetherness of the family. Placement of figures is around the table ${ }^{10}$ suggests need for attention. They all are eating dinner together shows the relationship has improved significantly. Absence of Elevation of figure (daughter) depicts improvement in her relationship. There is no line drawn at the bottom of page suggest stability in her family life. The need for love and warmth has taken a new representation in 
form of light bulb ${ }^{4 \& 7}$. It indicates that the client is still striving for love \& warmth. Absence of Flowers and trees suggests client's satisfaction about her spiritual development. In summary the third drawing reinforces several basic themes present in the first\& second drawings, but it also demonstrates an understandable change which was supported by client's verbal reports.

\section{Discussion}

The aim of this case report was to explore the role of KFD, bringing in insight/understanding in family dynamics and relationship of a female client and help her improve relationship with family members. The finding on the first drawing indicated that the client feels that there is relationship and communication issue in the family. The client feels unheard. The client is looking for some support in the family and she is not fully involved in life. Even though the client is saying that her relationship with daughter is good but the distance shown between them is contradicting her statement ${ }^{9}$. The findings were discussed with the client that helped her create insight and awareness about herself and the family which was taken up into the counselling. Family drawing assessments are an effective instrument for exploring more subjective, personal, and unconscious aspects of representational models of the self in relation to family ${ }^{13}$. The second drawing shows united family ${ }^{10}$. The struggle for love and warmth was resolved by the client by getting into religious practices ${ }^{11}$. Religious practises have made the client emotionally stable to handle her conflicts. Some new elements like flowers and trees were added in drawing indicating client's growth ${ }^{11}$. The client has used Compartmentalization to create boundaries by separating the family figures through curtain lining ${ }^{8}$. Finding on the third drawing shows the client can manage a much better relationship with her husband and daughter. There is lots of laughter and Happiness in the family, which was indicated by the picture of family having dinner together ${ }^{10}$. Presence of encapsulation in the third drawing suggests the client still feeling suffocated and she needs boundary drawn among the family members ${ }^{9}$. She wants family members to take up responsibility of their own life. This case study suggests that KFD resulted in insight building and conflict resolution among family members. Information found in KFD combined with clinical interviews, proved invaluable in setting therapeutic goals with the client, repeated themes and focal points suggest deep-rooted conflict and anxiety.

\section{Conclusion}

Diagnostic procedures such as the KFD offer easy and accurate access to information regarding attitudes and conflicts within the family. We believe that the KFD can be used as s a valuable diagnostic tool to understand the intervention in more depth. The client indicated that she became more tolerant and accepting towards herself and family members. She noted a softened attitude towards herself and her complaints towards the end of art therapy.

Funding: The source of funding is self.

Declaration of conflicting interests: The author declares that there is no conflict of interest. The conflict of interest is nil.

Ethical Clearance: Ethical clearance is taken from Center for Academic Research (CARE) Annamalai University, Psychology wing.

\section{References}

1. Cherry K, Morin A. How cognitive biases influence how you think and act. Verywell Mind. 2019.

2. Vazquez SS. Using Art Therapy to Express Your Self.

3. By CA, Narayan D. Paper Title: Arts Based Therapy to Create Empathetic, Inclusive Learning Spaces: An Eastern Perspective

4. Burns RC, Kaufman SH. Actions, styles and symbols in kinetic family drawings: An interpretative manual. Brunner/Mazel; 1972.

5. Burns RC. Self-growth in families: Kinetic Family Drawings (KFD) research and application. Brunner/ Mazel; 1982.

6. Schacker, Edward Henry, "The Kinetic Family Drawings As An Indicator of Marital Instability And Family Stress" (1979).

7. Anderson, Lynn Ann. A Comparison of Kinetic Family Drawings of Firstborn and Second-Born Siblings. May 2004, v + 228, Donna J. Habenicht

8. Wilkinson S. Drawing up boundaries: a technique. Journal of family therapy. 1985; 7(2):99-111.

9. Foley YC, Mullis F. Interpreting Children's Human Figure Drawings: Basic Guidelines for School Counselors. Georgia School Counselors Association Journal. 2008; 1(1):28-37. 
10. Travers RB. Critically aligning kinetic family drawing test protocol interpretations with existing family structures: A multiple case study from the Itsoseng clinic files (Doctoral dissertation, University of Pretoria). (Doctoral dissertation, University of Pretoria).

11. Monttinen, Anne-Maria J., "A Descriptive Study of the Kinetic Family Drawings of Children from
Catholic, Lutheran, and Seventh-day Adventist Religious Backgrounds"

12. Siegel IM, Kornfeld MS. Kinetic family drawing test for evaluating families having children with muscular dystrophy. Physical therapy. 1980 Mar 1; 60(3):293-8.

13. Lohn T. Schema of Family: A Developmental Comparison of Kinetic Family Drawings. 